\section{[gw22-e0907] LONG-TERM LIQUOR INTAKE IS AN INDEPENDENT RISK FACTOR OF INCIDENT HYPERTENSION IN MEN IN NORTH CHINA: A POPULATION-BASED COHORT STUDY}

Xiong-jing Jiang' ${ }^{1}$ Chen Jin², Shou-Ling Wu², Meng Peng ${ }^{1}$, Wei Ji', Yu-qing Zhang', Li-sheng Liu' ${ }^{1}$ Fuwai hospital, Beijing, China; ${ }^{2}$ Kailuan hospital, Tangshan, Hebei, China

10.1136/heartjnl-2011-300867.292

Background Heavy alcohol intake increases the risk of incident hypertension, but the relationship between light-to-moderate alcohol consumption and incident hypertension remains controversial. Liquor is very popular among men in north china. This study examined the association between liquor consumption and incident hypertension in men in north china during 4 years of follow-up.

Methods Kailuan study is a population-based cohort study for cardiovascular risk prevention, which enrolled 101510 employees (men 81 110) from the Kailuan coal group in Tangshan city in north China from June 2006 to September 2007. The authors prospectively followed 32389 male subjects from the KaiLuan Study, who were free of hypertension, cardiovascular diseases and cancer at baseline. All subjects performed routine medical examination once every 2 years and were followed up. Self-reported lifestyle, follow-up clinical data and questionnaires were collected. Total alcohol intake was summed from liquor.

Results During 4 years of follow-up, 9151 men developed hypertension (defined as new physician diagnosis, antihypertensive treatment, plus on-site measured systolic blood pressure $>=140 \mathrm{~mm} \mathrm{Hg}$ or diastolic blood pressure $>=90$ $\mathrm{mm} \mathrm{Hg}$ ). The 4 years incidence of hypertension for never, 1 to 50,50 to 100,100 to 150 and $>=150 \mathrm{~g}$ alcohol per day were $25.03 \%, 28.93 \%, 37.00 \%, 40.06 \%$ and $42.41 \%$, respectively. The authors found that alcohol intake was positively and significantly associated with the risk of incident hypertension. After adjustment by age and lifestyle, the risk ratio for incident hypertension increased linearly with alcohol consumption, compared with no alcohol intake. Adding potential intermediates (body mass index, diabetes and high cholesterol and family history of hypertension) this trend did not change. Models stratified by baseline systolic blood pressure $(<120,>=120 \mathrm{~mm} \mathrm{Hg}$ ) or diastolic blood pressure ( $<80 \mathrm{vs}>=80$ $\mathrm{mm} \mathrm{Hg}$ ) did not alter the relative risks. 
Conclusion In this north China population, long-term liquor intake is an independent risk factor of incident hypertension. Even light-to-moderate liquor consumption increased incident hypertension risk in men. 